\title{
Research on flow patterns of tourists in scenic spots based on Data Mining
}

\author{
Zhao Yingju \\ School of economics and management, Beijing Jiaotong University, Beijing, China
}

\begin{abstract}
For the fixed tourist routes in the scenic spot, the longer the journey is, the slower the speed is, and the easier the congestion is. This study is an exploratory study. In this paper, Yudaokou grassland forest scenic area, a nature reserve crossed by national No.1 scenic road, is selected as the research object. Based on the point- axis gradual diffusion theory, the mobile app is used to record the travel process of tourists on the same tourist route in the same scenic area, so as to calculate the travel speed of tourists on different road sections, and then predict the future congestion of the scenic area, the smaller the speed, the greater the probability of congestion; the greater the speed, the less time, the smaller the probability of congestion. Finally, the paper discusses the significance and theoretical contribution of the study on the two aspects of tourists' moving behavior and moving mode in scenic spots.
\end{abstract}

\section{Introduction}

In the humanistic urban planning concept, it is emphasized that the correct spatial planning should be to adapt to and meet human needs ${ }^{[1-2]}$. This concept returns to the tourism spatial design and product planning, and emphasizes that the main body of tourism activities "tourists" as the basic starting point, and the production and supply process of tourism products should introduce the consideration and evaluation of tourists' behavior ${ }^{[3-4]}$. Kurt Lewin, an American social psychologist, expressed the relationship between human and environment as a function. He believed that behavior is determined by the individual and his environment, i.e. $\mathrm{B}=\mathrm{f}(\mathrm{P} \cdot \mathrm{E})$; where $\mathrm{B}$ represents the individual's behavior, $\mathrm{P}$ represents an individual's internal conditions and characteristics, and e represents the individual's external environment. His behavior model reveals the general law of human behavior to some extent, that is, human behavior is the product of interaction between individuals and their environment ${ }^{[5]}$. Tourism flow is the basis of tourism and one of the core issues of tourism geography. The study of tourism flow pattern is an important aspect of tourism flow study. It is very important for us to study the flow pattern of tourism to reveal the law of tourism flow, understand the relationship between tourism destination and tourism flow, guide the practice of tourism development and implement the concept of sustainable tourism development. Use mobile app to record the tourists' moving track in the scenic spot, and record the corresponding time interval. Mobile data based on mobile users has the following characteristics: (1) mobile location can collect real location and mobile data of individuals, including time Multiple dimensions of space
[6]; (2) mobile data has higher quality and accuracy than traditional mobile data, and the coverage of mobile signal is wide, which greatly reduces the probability of no signal phenomenon; (3) real-time mobile information of individuals can be obtained based on mobile location ${ }^{[7]}$, which supports the analysis of individual spatiotemporal behavior trajectory; (4) mobile data is suitable for mobility measurement and modeling, The communication service system itself has certain mobile computing and communication analysis capabilities, which can reduce the information loss in the process of behavioral data coding, and is suitable for large-scale spatiotemporal data analysis ${ }^{[8]}$. Mobile data can overcome many shortcomings of traditional spatiotemporal data, and it is an important means to acquire individual spatiotemporal behavior data in the future. Under the premise of fixed time budget and limited mobile capacity, the space reach scope of tourism activities is limited. The space of tourism products and services is not mobile, which directly affects the use and evaluation of products and services ${ }^{[9]}$. Through the actual spatial behavior characteristics and flow patterns of tourists, the potential spatial behavior and flow patterns of tourists are predicted. Based on the needs of tourists, the matching between supply and demand is realized, so as to optimize the tourism experience of tourists.

The nature reserve refers to the land, land water area or sea area where the representative natural ecosystem, the natural concentrated distribution of rare and endangered wild animal and plant species, and the natural relics with special significance are located, and the area for special protection and management is designated according to law.

Nature reserves are a general term. In fact, there are many types of nature reserves due to different purposes, 
requirements and conditions. According to the main objects of protection, the nature reserves can be divided into three categories: ecosystem type, biological species and natural heritage; according to the nature of the nature of the nature of the nature reserves, the nature reserves can be divided into four categories: scientific research reserves, national parks (i.e. scenic spots), management areas and resource management reserves. Different from urban planning, there is less room for transformation in the specific space area of tourism product planning, such as World Heritage level tourism resources or nature reserves. Therefore, the traditional methods of tourism product planning focus on the evaluation, analysis and utilization of tourism resources from the very beginning. The process of production and supply of tourism products is actually the process of transformation and utilization of tourism space-time environment. No matter how small the transformation space of tourism product planning space is, it is against the people-oriented planning idea to ignore or desalinate "tourists" as the main body of tourism activities in this process ${ }^{[10]}$.

Taking the nature reserve as the research object, this paper uses the recording function of mobile app to calculate the travel mode and speed of tourists, and predict the future congestion of the scenic spot, so as to provide a scientific basis for the road planning of the scenic spot.

\section{Theoretical basis}

\section{1 point -axis gradual diffusion theory}

"Point axis" gradual diffusion theory is a complete theory put forward in 1984 by Lu Da Dao, a famous economic geographer in China, based on the central place theory, spatial diffusion theory and growth pole theory.

The "point" refers to the tourism node, including tourist attractions such as tourist attractions, tourist hotels and other tourist reception facilities, central towns at all levels of tourism and other tourism entities. Tourism node is not only the gathering point of all levels of tourism regions, but also the tourism center that drives the development of regional tourism. "Axis" is mainly composed of trunk lines and channels at all levels, such as traffic trunk lines, etc., which drives the circulation and flow of various resources such as information flow and material flow, realizes the effective circulation of various resources and the rational allocation of resources, and has strong economic attraction and cohesion for the nearby areas. The core of the theory is the summary of the theoretical model of "the best structure and the best development" of the region ${ }^{[11]}$.

\section{2 point -axis distribution of Yudaokou grassland forest scenic spot}

As shown in Figure 1, in the road network of Yudaokou grassland forest scenic spot, the big circle points represent the core attraction, the small circle points represent the auxiliary facilities, and the curve represents the traffic route of the tourism passing through the scenic spot and connecting each core scenic spot and auxiliary facilities. According to the point axis theory, large and small dots represent "points" in the road network system of scenic spots, and the connected route is "axis".

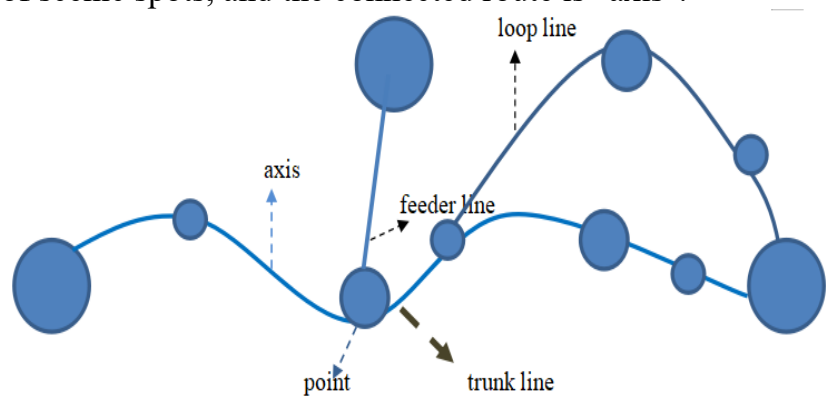

Figuer1. Point -axis distribution map of Yudaokou National Forest Park

\section{Research design}

\section{1 research question}

Taking Yudaokou grassland forest scenic spot as the research object, this paper uses the recording function of mobile app to calculate the travel mode and speed of tourists, the actual spatial behavior characteristics and flow mode of tourists, predict the potential spatial behavior and flow mode of tourists, realize the matching of supply and demand, optimize the layout of scenic spots and improve the tourism of tourists based on the needs of tourists experience.

\subsection{Research hypothesis}

1) The lower the speed, the more likely the congestion will occur: When the axis is fixed and the distance is the same, compare the speed of volunteers at points A1, A2, A3 and A4. If the speed of each point is smaller, it is easier to release congestion at the change point

2) The longer the time is, the more likely the congestion will occur: When the axis is fixed and the speed is equivalent, compare the time when volunteers arrive at points $\mathrm{A} 1, \mathrm{~A} 2, \mathrm{~A} 3$ and $\mathrm{A} 4$. If the time is longer when they arrive at a certain point, the easier the congestion will be released at the change point.

\subsection{Research methods}

This study intends to use case study method and statistical analysis method of action research.

\subsubsection{Exploratory research}

\subsubsection{1. method 1: calculation based on speed:}

Select the Yudaokou grassland forest scenic spot of the nature reserve for the experiment, and select volunteers with comparable physical strength, let them hold mobile phones with positioning function app, turn on the 
positioning to record their position and action track, and ensure that they do not deviate from the route, as shown in Figure 2.

Select points along the scenic spot with the same distance, assuming A1, A2, A3, A4 An where the distance between two adjacent points is equal and $\mathrm{S}$; at the same time, turn on the mobile phone timer, and assume that when each point is reached, the time is T1, T2, T3, T4 TN is shown in Figure 2.

Calculate the speed of two adjacent points, for example, the average speed from point a to point A1 is $\mathrm{s} /$ $\mathrm{t} 2-\mathrm{t} 1$. The average velocity from A2 to A3 is $\mathrm{s} / \mathrm{t} 3-\mathrm{t} 1$.

\subsubsection{2. calculation based on time proportion:}

Select the Yudaokou grassland forest scenic spot of the nature reserve for the experiment, and select volunteers with comparable physical strength, let them hold mobile phones with positioning function app, turn on the positioning to record their position and action track, and ensure that they do not deviate from the route, as shown in Figure 3.

Select points along the scenic spot with the same distance, assuming A1, A2, A3, A4 An if the distance between two adjacent points is equal, the proportion of the distance between two adjacent points to the total distance is $1 / \mathrm{n}-1$; at the same time, turn on the mobile phone timer, and assume that when each point is reached, the time is T1, T2, T3, T4 TN is shown in Figure 3.

Calculate the proportion of time taken by two adjacent points to the total time. For example, the proportion of time taken by two points a and $\mathrm{B}$ to the total time is $\mathrm{t} 2-\mathrm{t} 1 / \mathrm{t} 4-\mathrm{t} 1$, and the time taken by two points $\mathrm{B}$ and $\mathrm{C}$ is $\mathrm{t} 3-\mathrm{t} 2 / \mathrm{t} 4-\mathrm{t} 1$. The larger the calculated increment is, the greater the probability of congestion in this section will be, and it should be improved in time.

\subsection{2 validation study}

Exploratory research is based on the point axis distribution of scenic spots, select fixed axis direction and points on the axis to test, in order to verify its feasibility, put forward a confirmatory research.

\subsubsection{1 methods 2: calculation based on time:}

In two teams, select several groups of volunteers, mobile phones with positioning function app, make them enter from A1 port and come out from A4 port, open positioning to record their position and action track, and ensure that they do not deviate from the route. Volunteers can move freely on the internal point axis. At the end of the experiment, the collected tracks of volunteers were observed and analyzed.

\subsubsection{Research hypothesis:}

Suppose that one of the two groups of volunteers in several groups is a and B respectively, the following assumptions can be made:
If a and B stay at a certain point for more than 30 minutes and keep close to each other, and keep the distance within 5 meters, congestion will occur at that point;

If a and B stay at a certain point for more than 30 minutes, but the distance is long and close, and the distance always fluctuates in the range of 5-15 meters, then there is a resource point at this point, where tourists punch in here;

If a and B stay at a certain point for more than 30 minutes, a will always keep $b$ fluctuating in the range of 5-15 meters, while B is still, then there is a service facility point at the changed point, such as public toilets, where B will receive services.

Compare the research hypothesis of exploratory research with that of confirmatory research, stack on the point axis distribution map of the scenic spot, screen the points with high stacking degree, (newly discovered points), draw the congestion map of the scenic spot, and grasp the overall flow pattern of tourists in the scenic spot.

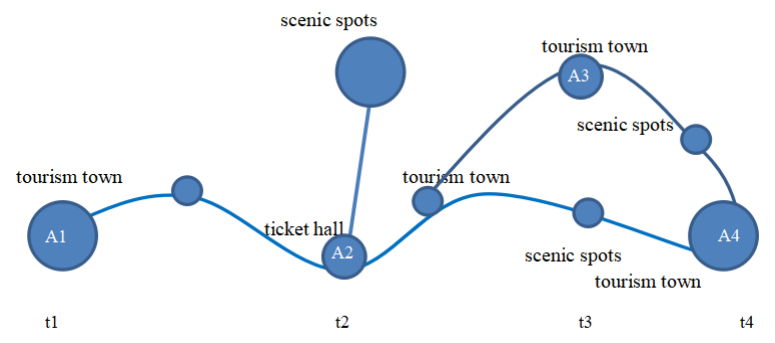

Figure 2. Speed based research diagram

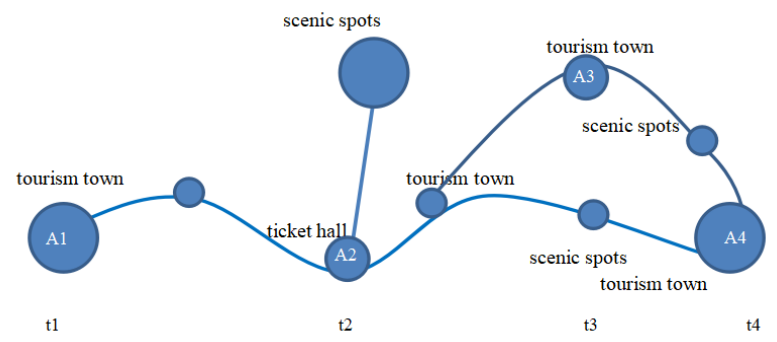

Figure 3. Time based research diagram

\section{4 suggestions and Countermeasures}

\section{1 guidance and navigation}

Tourists are mainly engaged in tourism activities to relax their mood and have leisure and entertainment. Therefore, they have poor initiative in obtaining information on the way to travel. This is that before tourists enter the scenic area, the scenic area should remind them to use mobile phone navigation on the guidance system to check the road conditions in front of them in real time. For example, this function can be realized by the mobile navigation app gothenko map. It is seriously blocked in red, slow driving in yellow, road construction in gray and smooth road in green. During the peak season of tourism, the traffic situation of the scenic spot can be predicted in real time through the electronic large screen at the 
intersection with branch lines, and no tourists can provide alternative routes.

\section{2 online ticket sales}

Based on the scientific assessment of the capacity of the scenic spot, the online ticket pre-sale method can be adopted. In addition, the online ticket sale method can be adopted according to the Gongwangfu scenic spot in Beijing. According to the number of tickets already sold, the number of tourists entering the scenic spot can be controlled.

\section{3 construction of tourism transportation network}

According to the previous road congestion situation, select the key blocked road sections, and establish branches around the blocked road sections that diverge from the main line, open the traffic capillaries, create a closed-loop traffic network structure, and prevent the scenic area from blocking.

\section{4 opening by time division}

According to the flow monitoring data of tourists in different time periods and different areas, the timesharing and district opening is implemented. For places where people gather easily, visitors should be arranged to enter the park in an interval way. In the entrance and exit of the scenic spot, important tourist spots and other places where people are likely to gather, management personnel shall be provided to strengthen the order management of tourists, so as to optimize the setting of tourist routes.

\section{Summary and discussion}

This paper takes Yudaokou grassland forest scenic spot as the research object, and uses the recording function of mobile app to calculate the traveling mode and speed of tourists, forecast the future congestion situation of the scenic spot: when the distance is certain, the less time and the greater speed, the less likely it is to be congested. On the contrary, it provides a scientific basis for the road planning of the scenic spot. But in this study, there are many shortcomings, some variables will affect the results. For example, due to the small number of people in the selected scenic spot, the speed of each road section is the same, and the correct result cannot be obtained. In the future, more complex mathematical models can be used to consider the impact of various factors on the congestion of scenic spots; different case sites can also be selected to increase the universality of the experiment; volunteers can be selected for empirical research, and then the results of comparative and exploratory research can be compared, and the research design can be improved according to the results.

\section{Acknowledgment}

After continuous efforts and unremitting insistence, this paper has finally been completed. Although the length is small, it has not come easily. Looking back on the postgraduate's study experience in the past six months, I can't help but feel a thousand emotions and remember the past.

The completion of this paper benefits from the help of all parties. First of all, thank my teacher. From the determination of the topic, the formation of the research framework, the multiple revisions of the first draft to the final completion of the thesis, the teacher devoted himself to every step, gathering his hard work and wisdom, and each circle and revision is full of his good intentions. His rigorous and pragmatic attitude, profound knowledge, keen academic insight, selfless work enthusiasm and honest and modest personality charm deeply affected me. In the process of my thesis creation, the teacher is always full of hope for me, constantly urging and motivating me. When I feel confused and confused, I can always get accurate guidance from the teacher. His encouragement is the driving force for me to overcome difficulties. In the future, I will continue to work hard and forge ahead to keep up with the teacher's training.

Secondly, I would like to thank all the teachers of the college for their teaching, guidance and help in my study. Besides, thank you all. Thank you, elder martial sister, for your guidance and help in the process of thesis writing, for your encouragement and concern, and for your companionship along the way.

\section{Reference}

1. Duan Yifu. My opinion on human geography [J]. Progress of geographical science, 2006, (2): 1-7 .

2. Kang Yanhong, Zhang Jingxiang. Reflection on humanistic urban planning [J]. Journal of urban planning, 2006, (1): 56-59 .

3. Chai Yanwei, Tana. Research progress of temporal and spatial behavior in China $[\mathrm{J}]$. Progress in Geosciences, 2013, (9): 1362-1373.

4. Huang Xiaoting, Zhu Shuwei, Zhao Ying. Product following behavior: product planning method of tourism time [J]. Journal of tourism, 2016, (5): 36-44.

5. Jiang Lin. consumer behavior [M]. Beijing: Capital University of economics and trade press, 200: 1-150.

6. Jie Zhu. A study on the flow pattern of domestic tourism in Jiangxi Province [D]. Nanjing University, 2003.

7. Zook M, Dodge M. New Digital Geographies : Information, Communication and Place[M] / / Brunn S D, Cut t ter S L. Harrington J W. Geography and Technology. Nethedand: Kluwer Academic Publishefs, 2004.

8. Ahas R, Mark U. Location-based Services: New Challenges for Planning and Public 
Administration?[J] . Futures, 2005, 37 : 547561 .

9. Miller H J. What about People in Geography Information Science?[c] / / Fisher P, Unwin D. Representing GIS. New Jersey : John Wiley \& Sons . Ltd. 2005.

10. Zhao Ying, Wang Li, Huang Xiaoting, Tana. The influence of theme park performance project on tourists' activity space -- Based on the analysis of spatiotemporal accessibility [J]. Journal of tourism, 2017,32 (12): 49-57.

11. Chen Wenjie, Yang Guoliang, Huang rongjuan, Wen Liling. Strategic research on tourism spatial development of Beibu Gulf based on "point axis" gradual diffusion theory $[\mathrm{J}]$. Special Economic Zone, 2010 (07): 206-208. 